\title{
Association of Single-Nucleotide Polymorphisms in DC-SIGN with Nasopharyngeal Carcinoma Susceptibility
}

\author{
Sisi Li, ${ }^{1}$ Zhifang Lu, ${ }^{2}$ Mengwei Yao, ${ }^{2}$ Sisi Ning, ${ }^{2}$ Yuan $\mathrm{Wu}^{2}$ Xunzhao Zhou, ${ }^{2}$ \\ Changtao Zhong, ${ }^{2}$ Kui Yan, ${ }^{2}$ Ying Xie, ${ }^{3}$ and Zhengbo Wei ${ }^{1}$ \\ ${ }^{1}$ Department of Head and Neck Tumor Surgery, Affiliated Tumor Hospital of Guangxi Medical University, Nanning, China \\ ${ }^{2}$ Graduate School of Guangxi Medical University, Nanning, China \\ ${ }^{3}$ Guangxi Key Laboratory for High-Incidence Tumor Prevention and Treatment, Experimental Center of Medical Science of Guangxi \\ Medical University, Nanning, China
}

Correspondence should be addressed to Ying Xie; xieying2002@hotmail.com and Zhengbo Wei; wzhbo1973@aliyun.com

Received 2 February 2017; Accepted 8 May 2017; Published 14 June 2017

Academic Editor: Marco E. M. Peluso

Copyright (C) 2017 Sisi Li et al. This is an open access article distributed under the Creative Commons Attribution License, which permits unrestricted use, distribution, and reproduction in any medium, provided the original work is properly cited.

The aim of this study was to explore potential relationships of four single-nucleotide polymorphisms (SNPs) in the gene encoding dendritic cell-specific intercellular adhesion molecule 3-grabbing nonintegrin (DC-SIGN) with risk of nasopharyngeal carcinoma (NPC). The DC-SIGN SNPs rs7252229, rs4804803, rs2287886, and rs735240 were genotyped in 477 unrelated NPC patients and 561 cancer-free controls. At rs7252229, risk of NPC was significantly lower in individuals with GC (odds ratio [OR] 0.076, 95\% confidence interval [CI] 0.008-0.690), GG (OR 0.056, 95\%CI 0.006-0.487), or GC+ GG (OR 0.059, 95\%CI 0.007-0.515) than in individuals with the CC genotype, after adjusting for age, gender, smoking history, and EBV-VCA-IgA status. At rs4804803, risk of NPC was significantly higher in individuals with the genotype GG than in those with the genotype AA (adjusted OR 9.038, 95\%CI 1.708-47.822). At rs735240, risk of NPC did not change significantly with genotypes AG, GG, or AG + GG after adjusting for age, gender, and smoking history. However, when data were also adjusted for EBV-VCA-IgA status, three genotypes emerged as associated with significantly higher risk of NPC than the AA genotype: AG (OR 2.976, 95\%CI 1.123-7.888), GG (OR 3.314, 95\%CI 1.274-8.622), or GG + AG (OR 3.191, 95\%CI 1.237-8.230). Our results suggest that DC-SIGN SNPs rs7252229, rs4804803, and rs735240 may influence NPC risk in the Chinese population. The mechanisms mediating this risk require a further study.

\section{Introduction}

Nasopharyngeal carcinoma (NPC) is one of the most common malignant tumors in southern China, especially Guangdong and Guangxi provinces. In these endemic areas, annual NPC incidence reaches $20-30$ per 100,000 [1]. The primary risk factors for NPC include Epstein-Barr virus (EBV) infection, environmental carcinogens, and certain ethnic backgrounds [2].

EBV infection is strongly associated with NPC pathogenesis [3], and approximately $98 \%$ of all NPC cases are EBVrelated [4]. EBV may help drive NPC by encoding several oncogenic proteins, such as LMP1, which transform infected epithelial cells. In addition, the virus helps transformed cells evade host immune responses $[5,6]$. One protein involved in this immune evasion may be dendritic cell-specific intercellular adhesion molecule 3-grabbing nonintegrin (DC-SIGN), a type II membrane protein of the C-type lectin receptor superfamily [7] that is encoded by CD209 on chromosome 19p13.3. Expressed mainly on the surface of immature dendritic cells (DCs), macrophages, and B lymphocytes, DC-SIGN recognizes and induces interactions with many pathogens, including bacteria, virus, and parasites [8-13]. DC-SIGN on immature DCs can bind carcinoembryonic antigen (CEA), inhibiting DC maturation and thereby inducing tolerance of tumor cells [9]. The SKBR3 
breast carcinoma cell line can interact with DC-SIGN on macrophages to induce interleukin-10 secretion, contributing to an immunosuppressive environment [14].

Several single-nucleotide polymorphisms (SNPs) in the DC-SIGN gene have been associated with elevated risk of human diseases such as tuberculosis, dengue, AIDS, and cancer. For example, the SNP rs2287886 in the area of the promoter and SNP rs7248637 in the $3^{\prime}$-untranslated region may be associated with susceptibility to colorectal carcinoma [15]. The SNPs rs2287886, rs735240, and rs735239 correlated with NPC risk in a Cantonese population from Guangdong [16], while rs 4804800 and rs7248637 correlated with risk in a North African population [10].

In the present study, we investigated the possible genetic associations of the DC-SIGN SNPs with NPC risk in a Chinese population in the NPC-endemic Guangxi province of China.

\section{Methods}

2.1. Study Participants. The study was approved by the Ethics Committee of Guangxi Medical University. Unrelated NPC patients who were diagnosed with NPC and treated between July 2012 and June 2015 at the Affiliated Tumor Hospital of Guangxi Medical University (Guangxi, China) were enrolled. In addition, 561 healthy controls were recruited among those undergoing routine physical examinations at the Affiliated Tumor Hospital and the First Affiliated Hospital of Guangxi Medical University. The selection criteria for the healthy controls included the following: (1) no individual history of malignant tumors, (2) undergoing a health examination during the same enrollment period as the NPC cases, and (3) a local resident of Guangxi province. All study participants provided written informed consent.

2.2. Blood Collection. Peripheral venous blood $(3 \mathrm{~mL})$ was drawn from all subjects into EDTA-containing tubes, and genomic DNA was isolated using the TGuide Blood Genomic DNA Kit (Tiangen, Beijing, China) according to the manufacturer's instructions. DNA quality was evaluated using agarose gel electrophoresis. Samples of verifiable quality were stored at $-20^{\circ} \mathrm{C}$.

2.3. Selection of SNPs. A total of 4 SNPs (rs7252229, rs2287886, rs4804803, and rs735240) within DC-SIGN were selected to the following analyses based on the following selection strategy: (1) Using the 1000 Genomes Browser (http://phase3browser.1000genomes.org/index.html) and the NCBI database (http://www.ncbi.nlm.nih.gov), SNP files of DC-SIGN were obtained for the Beijing Han Chinese (CHB) population. (2) Using Haploview 4.2 software, the SNP files were analyzed and the tagging SNPs which met the below criteria were selected: (i) minimum allele frequency (MAF) of greater than $5 \%$ in the $\mathrm{CHB}$ population; (ii) location within the coding region or the promoter region or the $3^{\prime}$ - or $5^{\prime}$-untranslated regions (UTRs); and (iii) no strong linkage disequilibrium (LD; $\left.r^{2}<0.80\right)$ with any other selected SNPs. (3) The potential function of the tagging SNPs was analyzed using SNP function prediction in the website http://snpinfo.niehs.nih.gov/snpinfo/snpfunc.htm. (4) We also forced the inclusion of some SNPs which did not fulfill the above selection criteria, for their functionality has been indicated in published studies. (5) Compatibility of selected SNPs through the above steps with the platform of MALDI-TOF mass spectrometry-based genotyping was analyzed by the BGI company (Beijing, China), which provided the SNP genotyping support. And the SNPs which were not technically compatible with the genotyping platform were excluded from the following formal genotyping.

2.4. Mass Spectrometry-Based Genotyping. The DC-SIGN SNPs rs7252229, rs4804803, rs2287886, and rs735240 were genotyped in all subjects as described [17] using allelespecific matrix-assisted laser desorption/ionization time-offlight (MALDI-TOF) mass spectrometry, with support from BGI (Beijing, China). Fragments spanning the SNPs were amplified by PCR using primers designed with MassARRAY Assay Design 3.1 Software (Sequenom, San Diego, CA, USA). Amplifications were conducted in a 384-well ABI Veriti PCR System (Applied Biosystems) following the manufacturer's instructions. Amplification reactions $(5 \mu \mathrm{L})$ contained $4 \mu \mathrm{L}$ Master Mix and $1 \mu \mathrm{L}$ DNA $(20 \mathrm{ng} / \mu \mathrm{L})$, and reaction conditions were as follows: $94^{\circ} \mathrm{C}$ for $5 \mathrm{~min}$, followed by 45 cycles of $94^{\circ} \mathrm{C}$ for $20 \mathrm{sec}, 56^{\circ} \mathrm{C}$ for $30 \mathrm{sec}$, and $72^{\circ} \mathrm{C}$ for $1 \mathrm{~min}$. Alleles were analyzed using MassARRAY TYPER 4.0 software (Sequenom). Successful genotyping rates were 99.8\% (560/561) in the control group for rs7252229, rs4804803, and rs735240, respectively, and were $99.8 \%(476 / 477)$ in the NPC group and $99.6 \%(559 / 561)$ in the control group for $\mathrm{rs} 2287886$.

2.5. Statistical Analysis. Data were analyzed using SPSS 17.0 (IBM, Armonk, NY, USA), and the threshold for significance was defined as two-sided $P<0.05$. NPC patients and healthy controls were compared in terms of gender composition, smoking history, and the presence of immunoglobulin $\mathrm{A}$ against EBV capsid antigen (EBV-VCA-IgA) using the chi-squared test. The ages of patients and controls were compared using an unpaired $t$-test. Genotype distributions in healthy controls were compared against the predictions of Hardy-Weinberg equilibrium (HWE) using the Pearson chi-squared test. Genotype frequencies were compared between patients and controls using the chi-squared test. Possible associations between DC-SIGN SNP genotypes and risk of NPC were identified using logistic regression; odds ratios (ORs) with 95\% confidence intervals (CIs) were calculated when appropriate. Unconditional multivariate logistic regression was used to calculate adjusted ORs and $95 \%$ CIs after adjusting for age, gender, smoking history, and EBV-VCA-IgA status, unless otherwise noted. The goal was to eliminate possible effects of these confounding factors in order to isolate the effects of each SNP on NPC risk.

\section{Results}

3.1. Characteristics of Patients and Controls. A total of 477 NPC patients and 561 healthy controls were included in our study. While the two groups were similar in 
age $(P=0.056)$, they differed significantly in gender composition $(P<0.001)$, smoking history $(P<0.001)$, and EBVVCA-IgA status $(P<0.001$; Table 1$)$. Frequencies of each SNP genotype were in agreement with $\mathrm{HWE}$ in the control population: rs7252229, $P=0.307$; rs4804803, $P=0.533$; rs2287886, $P=0.331$; and rs735240, $P=0.937$.

3.2. Correlation of rs 7252229 Polymorphism with Risk of NPC. Frequency of the genotype GG + GC at rs7252229 was significantly lower in NPC patients than in healthy controls (0.18\% versus $1.68 \%)$, corresponding to an unadjusted OR of 0.105 (95\%CI $0.013-0.842)$ and an adjusted OR of 0.059 (95\%CI 0.007-0.515; Table 2). The genotypes GC and GG were also associated with lower risk than the genotype CC: the respective unadjusted ORs were 0.111 (95\%CI $0.014-0.914)$ and 0.104 (95\%CI $0.013-0.834)$; the corresponding adjusted ORs were $0.076(0.008-0.690)$ and 0.056 (95\%CI $0.006-0.488$ ).

3.3. Correlation of rs2287886 Polymorphism with Risk of NPC. Relative to individuals with the genotype AA, individuals with the genotype $A G$ or $G G$ were at similar risk of NPC based on unadjusted and adjusted OR calculations (Table 3).

3.4. Correlation of rs4804803 Polymorphism with Risk of $N P C$. Individuals with the genotype GG were at significantly higher risk of NPC than those with the genotype AA (Table 4). The unadjusted OR was 4.800 (95\%CI 1.014-22.732) and the adjusted OR was 9.038 (95\%CI $1.708-47.822)$.

3.5. Correlation of rs735240 Polymorphism with Risk of NPC. Frequencies of genotypes AA, AG, and GG did not differ significantly between patients and controls before or after adjusting for age, gender, and smoking history. Interestingly, after controlling for EBV-VCA-IgA status, the genotypes GG, AG, and GG + AG emerged as conferring higher risk of NPC, with respective ORs of 2.976 (95\%CI 1.123-7.888), 3.314 (95\%CI 1.274-8.622), and 3.191 (95\%CI 1.237-8.230) (Table 5). Next, we compared genotype frequencies between patients and controls in separate subgroups among all subjects negative for EBV-VCA-IgA and among all subjects positive for EBV-VCA-IgA. Among those negative for EBV-VCA-IgA, the genotypes GG, AG, and GG + AG were significantly more frequent among patients than controls, with respective ORs of 8.797 (95\%CI 1.175-65.838), 2.077 (95\%CI 1.055-4.087), and 8.710 (95\%CI $1.168-$ 64.940). Among subjects positive for EBV-VCA-IgA, in contrast, genotype frequencies were similar between patients and controls.

\section{Discussion}

Our data showed significantly lower frequency of the GC + GG genotypes at rs7252229 in NPC patients than in healthy controls, suggesting that the genotype CC increases risk of NPC. This appears to be the first report linking rs7252229 with risk of malignant disease. Another study linked the C allele at rs7252229 with elevated risk of invasive
TABLE 1: Clinical characteristic of Chinese patients with NPC and cancer-free controls.

\begin{tabular}{lcccc}
\hline Subgroup & Cases & Controls & $\chi^{2} / t$ & $P$ \\
\hline$N$ & 477 & 561 & & \\
Gender, $n$ & & & 42.121 & $<0.001^{\mathrm{a}}$ \\
$\quad$ Male & 365 & 322 & & \\
$\quad$ Female & 112 & 239 & & \\
Age, yr & $46.68 \pm 11.60$ & $48.17 \pm 13.53$ & 1.912 & $0.056^{\mathrm{b}}$ \\
EBV-VCA-IgA & & & 197.811 & $<0.001^{\mathrm{a}}$ \\
status, $n$ & & & & \\
$\quad$ Positive & & 15 & & \\
$\quad$ Negative & 314 & 546 & & \\
Smoking history, $n$ & 163 & & 21.883 & $<0.001^{\mathrm{a}}$ \\
$\quad$ Yes & 163 & 119 & & \\
$\quad$ No & 314 & 442 & & \\
\hline
\end{tabular}

${ }^{\mathrm{a}}$ Chi-squared test; ${ }^{\mathrm{b}}$ two-sample $t$-test. EBV-VCA-IgA: immunoglobulin A against Epstein-Barr virus capsid antigen; NPC: nasopharyngeal carcinoma; OR: odds ratio.

pulmonary aspergillosis [11]. How rs7252229 may contribute to NPC pathogenesis is unclear. Since rs7252229 lies within an intron in the DC-SIGN gene and it is predicted to be a binding site for some transcripts (based on http://snpinfo. niehs.nih.gov/cgi-bin/snpinfo/snpfunc.cgi), we predict that the genotype CC at rs7252229 may affect DC-SIGN expression and thereby overall activity. It is also possible that rs7252229 polymorphism affects humoral and cellmediated immunity: in a study of healthy school children and young adults who received the measles vaccine, the genotype CC was associated with higher specific neutralizing antibody titers than the genotype GG among AfricanAmericans, while the genotype CC was associated with lower interferon- $\gamma$ levels than genotypes GG and GC among Caucasians [12]. Thus, it is plausible that this SNP might be associated with immunological abnormality in NPC patients as well as immune escape by EBV-infected NPC cells. This should be investigated in future studies.

Our data showed a significantly higher frequency of the genotype GG at rs4804803 in patients than in controls, indicating that this DC-SIGN SNP is associated with elevated NPC risk. Our results are inconsistent with those of studies in a Cantonese population [16] and a North African population [10] that found no relation between this SNP and NPC risk. Two possible explanations for this discrepancy are that (1) differences in genetic background and linkage disequilibrium between our population of mostly Zhuang minority subjects and the Cantonese subjects in the previous study [16] may mean different effects of DC-SIGN polymorphism on NPC risk and/or (2) DC-SIGN alleles are present at different frequencies across populations. Indeed, the frequency of the $G$ allele in our control group was $8 \%(82 / 1038)$, much lower than the frequencies of approximately $26 \%$ reported for Caucasian and North African populations [10, 13]. How the SNP rs4804803 may influence NPC risk is uncertain. It is located only $214 \mathrm{bp}$ upstream from the major Sp1-like binding region. We speculate that polymorphism in this 
TABLE 2: Frequencies of genotypes at DC-SIGN rs7252229 in NPC patients and healthy controls.

\begin{tabular}{|c|c|c|c|c|c|c|c|}
\hline Genotype & Cases & Controls & Unadjusted OR (95\%CI) & $P$ & Adjusted $\mathrm{OR}^{*}(95 \% \mathrm{CI})$ & Adjusted $P$ & $P_{\mathrm{HWE}}$ \\
\hline \multicolumn{7}{|l|}{ rs7252229 } & \multirow{5}{*}{0.307} \\
\hline CC & 8 & 1 & 1.000 & & 1.000 & & \\
\hline GC & 65 & 73 & $0.111(0.014-0.914)$ & 0.041 & $0.076(0.008-0.690)$ & 0.022 & \\
\hline GG & 404 & 486 & $0.104(0.013-0.834)$ & 0.033 & $0.056(0.006-0.487)$ & 0.009 & \\
\hline $\mathrm{GC}+\mathrm{GG}$ & 469 & 559 & $0.105(0.013-0.842)$ & 0.034 & $0.059(0.007-0.515)$ & 0.010 & \\
\hline
\end{tabular}

${ }^{*}$ Calculated using multiple logistic regression after controlling for age, sex, smoking history, and EBV infection factors. CI: confidence interval; DC-SIGN: dendritic cell-specific intercellular adhesion molecule 3-grabbing nonintegrin; HWE: Hardy-Weinberg equilibrium; NPC: nasopharyngeal carcinoma; OR: odds ratio.

TABLE 3: Frequencies of genotypes at DC-SIGN rs2287886 in NPC patients and healthy controls.

\begin{tabular}{ccccccr}
\hline Genotype & Cases & Controls & Unadjusted OR (95\%CI) & $P$ & $\begin{array}{c}\text { Adjusted OR } \\
(95 \% \mathrm{CI})\end{array}$ & Adjusted $P$ \\
\hline rs2287886 & & & & & \\
AA & 236 & 294 & 1.000 & & 1.000 & 0.331 \\
AG & 202 & 229 & $1.099(0.851-1.418)$ & 0.469 & $1.174(0.778-1.772)$ & 0.444 \\
GG & 38 & 36 & $1.315(0.808-2.140)$ & 0.270 & $0.819(0.345-1.94)$ & 0.651 \\
\hline
\end{tabular}

${ }^{*}$ Calculated using multiple logistic regression after controlling for age, sex, smoking history, and EBV infection factors. CI: confidence interval; DC-SIGN: dendritic cell-specific intercellular adhesion molecule 3-grabbing nonintegrin; HWE: Hardy-Weinberg equilibrium; NPC: nasopharyngeal carcinoma; OR: odds ratio.

TABLE 4: Frequencies of genotypes at DC-SIGN rs4804803 in NPC patients and healthy controls.

\begin{tabular}{|c|c|c|c|c|c|c|c|}
\hline Genotype & Cases & Controls & Unadjusted OR (95\%CI) & $P$ & $\begin{array}{c}\text { Adjusted OR* } \\
(95 \% \mathrm{CI})\end{array}$ & Adjusted $P$ & $P_{\mathrm{HWE}}$ \\
\hline rs4804803 & & & & & & & 0.533 \\
\hline AA & 400 & 480 & 1.000 & & 1.000 & & \\
\hline AG & 69 & 78 & $1.062(0.748-1.506)$ & 0.738 & $1.269(0.803-2.005)$ & 0.307 & \\
\hline GG & 8 & 2 & $4.800(1.014-22.732)$ & 0.048 & $9.038(1.708-47.822)$ & 0.010 & \\
\hline $\mathrm{GG}+\mathrm{AG}$ & 77 & 80 & $1.155(0.822-1.623)$ & 0.406 & $1.447(0.934-2.241)$ & 0.098 & \\
\hline
\end{tabular}

${ }^{*}$ Calculated using multiple logistic regression after controlling for age, sex, smoking history, and EBV-VCA-IgA status. CI: confidence interval; DC-SIGN: dendritic cell-specific intercellular adhesion molecule 3-grabbing nonintegrin; HWE: Hardy-Weinberg equilibrium; NPC: nasopharyngeal carcinoma; OR: odds ratio.

TABLE 5: Frequencies of genotypes at DC-SIGN rs735240 in NPC patients and healthy controls.

\begin{tabular}{|c|c|c|c|c|c|c|c|c|c|}
\hline Genotype & Cases & Controls & Unadjusted OR (95\%CI) & $P$ & $\begin{array}{c}\text { Adjusted } \\
\mathrm{OR}^{*}(95 \% \mathrm{CI}) \\
\end{array}$ & Adjusted $P$ & $\begin{array}{c}\text { Adjusted } \\
\mathrm{OR}^{\wedge}(95 \% \mathrm{CI})\end{array}$ & Adjusted $P$ & $P_{\mathrm{HWE}}$ \\
\hline rs735240 & & & & & & & & & 0.937 \\
\hline AA & 24 & 28 & 1.000 & & 1.000 & & 1.000 & & \\
\hline AG & 161 & 193 & $0.973(0.543-1.745)$ & 0.927 & $1.044(0.574-1.901)$ & 0.887 & $2.976(1.123-7.888)$ & 0.028 & \\
\hline GG & 292 & 339 & $1.005(0.570-1.772)$ & 0.986 & $1.077(0.601-1.927)$ & 0.804 & $3.314(1.274-8.622)$ & 0.014 & \\
\hline $\mathrm{AG}+\mathrm{GG}$ & 453 & 532 & $0.993(0.568-1.738)$ & 0.982 & $1.065(0.600-1.891)$ & 0.830 & $3.191(1.237-8.230)$ & 0.016 & \\
\hline
\end{tabular}

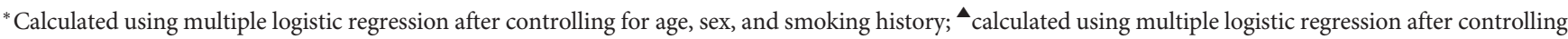
for age, sex, smoking history, and EBV-VCA-IgA status. CI: confidence interval; DC-SIGN: dendritic cell-specific intercellular adhesion molecule 3-grabbing nonintegrin; HWE: Hardy-Weinberg equilibrium; NPC: nasopharyngeal carcinoma; OR: odds ratio.

region affects DC-SIGN gene transcription and thereby its expression [16].

The SNP rs2287886, located close to the AP-1 binding site, was not associated with NPC risk in our study. While this result is consistent with the findings in a North African population [10], it contrasts with a previous report showing that Guangdong individuals carrying the $G$ allele at rs2287886 were at 1.42 -fold higher risk of NPC than those 
with the A allele [16]. This discrepancy may reflect differences in genetic background and linkage disequilibrium between our mostly Zhuang minority subjects and the Cantonese subjects in the Guangdong study.

We observed no significant relationship of rs735240 with risk of NPC based on unadjusted ORs or ORs adjusted for age, sex, and smoking history. However, we found that the frequency of the genotype AA was significantly lower in patients than in controls among subjects negative for EBVVCA-IgA. Thus, our results suggest that the genotype AA may protect against NPC in individuals negative for EBVrelated antibodies. This implies that the SNP rs735240 and EBV infection may interact to influence risk of NPC.

How this interaction occurs is unclear. EBV can infect DC-SIGN-positive cells including monocytes, immature DCs, and some macrophages $[16,18,19]$. Polymorphism in the DC-SIGN gene has already been shown to influence how easily DCs that are infected with cytomegalovirus, a herpesvirus with a structure similar to that of EBV: DCs from individuals with the genotype GG at rs735240 and the genotype AA at rs2287886 (GGAA) express higher levels of DC-SIGN and are more efficiently infected by cytomegalovirus than DCs carrying the AAGG genotype [20]. An analogous situation may exist for EBV, since glycoproteins on the viral surface, which are conserved with those on cytomegalovirus [21], appear to bind to DC-SIGN $[16,22,23]$ and allow the virus to enter B cells and epithelial cells [24]. This leads us to propose that the genotype AA at rs735240 protects against NPC by reducing DC-SIGN expression, making it more difficult for EBV to infect DCs and nasopharyngeal epithelial cells.

It is noteworthy in our study that the NPC and control group differed significantly in gender composition and smoking status (Table 1). Although these differences suggested that sex and smoking habit might be confounding factors affecting the results, no significant differences in our following analyses were showed in genotype frequencies at rs2887886 and rs4804803 and rs7252229 and rs735240 between males and females or ever-smokers and never-smokers in both NPC and control groups (detail not shown). Thus, it is unlikely that these SNPs vary with gender or smoking status. In any case, we controlled for sex, smoking status, and age by performing adjustment analyses using logistic regression, thereby eliminating or minimizing any possible confounding effects of these factors.

The results of the present study should be interpreted with caution given its important limitations. One is the relatively small sample, which increases the risk of selection bias. Another is our cross-sectional design, which means we cannot exclude the possibility that some of our controls will go on to develop NPC or other malignant tumors.

\section{Conclusion}

The present study identifies several SNPs in the promoter region of the DC-SIGN gene that may play an important role in NPC pathogenesis. The genotype CC at rs7252229 and the genotype GG at rs4804803 may be associated with elevated NPC risk, while the genotype AA at rs735240 may be associated with decreased NPC risk among individuals negative for EBV-VCA-IgA.

\section{Conflicts of Interest}

The authors declare no conflicts of interest.

\section{Authors' Contributions}

Sisi Li and Zhifang Lu contributed equally to this work.

\section{Acknowledgments}

This study was supported by the National Natural Science Foundation of China (81360406 and 81460415) and the Guangxi Natural Science Foundation of China (2014GXNSFAA118187 and 2016GXNSFAA380096).

\section{References}

[1] Y. Xie, Y. Wu, X. Zhou, M. Yao, S. Ning, and Z. Wei, "Association of polymorphisms hOGGI rs1052133 and hMUTYH rs3219472 with risk of nasopharyngeal carcinoma in a Chinese population," OncoTargets and Therapy, vol. 9, pp. 755-760, 2016.

[2] A. Edreis, M. A. Mohamed, N. S. Mohamed, and E. E. Siddig, "Molecular detection of Epstein - Barr virus in nasopharyngeal carcinoma among Sudanese population," Infectious Agents and Cancer, vol. 11, p. 55, 2016.

[3] A. Hildesheim and C. P. Wang, "Genetic predisposition factors and nasopharyngeal carcinoma risk: a review of epidemiological association studies, 2000-2011: Rosetta stone for NPC: genetics, viral infection, and other environmental factors," Seminars in Cancer Biology, vol. 22, no. 2, pp. 107-116, 2012.

[4] S. W. Tsao, C. M. Tsang, K. F. To, and K. W. Lo, "The role of Epstein-Barr virus in epithelial malignancies," The Journal of Pathology, vol. 235, no. 2, pp. 323-333, 2015.

[5] Y. Shen, S. Zhang, R. Sun, T. Wu, and J. Qian, "Understanding the interplay between host immunity and Epstein-Barr virus in NPC patients," Emerging Microbes \& Infections, vol. 4, no. 3, article e20, 2015.

[6] M. E. Ressing, M. van Gent, A. M. Gram, M. J. Hooykaas, S. J. Piersma, and E. J. Wiertz, "Immune evasion by Epstein-Barr virus," Current Topics in Microbiology and Immunology, vol. 391, pp. 355-381, 2015.

[7] F. Zhang, S. Ren, and Y. Zuo, "DC-SIGN, DC-SIGNR and LSECtin: C-type lectins for infection," International Reviews of Immunology, vol. 33, no. 1, pp. 54-66, 2014.

[8] T. B. Geijtenbeek, D. S. Kwon, R. Torensma et al., "DC-SIGN, a dendritic cell-specific HIV-1-binding protein that enhances trans-infection of T cells," Cell, vol. 100, no. 5, pp. 587-597, 2000.

[9] K. P. van Gisbergen, C. A. Aarnoudse, G. A. Meijer, T. B. Geijtenbeek, and Y. van Kooyk, "Dendritic cells recognize tumor-specific glycosylation of carcinoembryonic antigen on colorectal cancer cells through dendritic cell-specific intercellular adhesion molecule-3-grabbing nonintegrin," Cancer Reaserch, vol. 65, no. 13, pp. 5935-5944, 2005.

[10] K. Moumad, J. Lascorz, M. Bevier et al., "Genetic polymorphisms in host innate immune sensor genes and the risk of 
nasopharyngeal carcinoma in North Africa," G3 (Bethesda), vol. 3, no. 6, pp. 971-977, 2013.

[11] J. Sainz, C. B. Lupianez, J. Segura-Catena et al., "Dectin-1 and DC-SIGN polymorphisms associated with invasive pulmonary aspergillosis infection," PloS One, vol. 7, no. 2, p. e32273, 2012.

[12] I. G. Ovsyannikova, I. H. Haralambieva, R. A. Vierkant, M. M. O'Byrne, R. M. Jacobson, and G. A. Poland, "The association of CD46, SLAM and CD209 cellular receptor gene SNPs with variations in measles vaccine-induced immune responses: a replication study and examination of novel polymorphisms," Human Heredity, vol. 72, no. 3, pp. 206-223, 2011.

[13] M. A. Portman, H. W. Wiener, M. Silva, A. Shendre, and S. Shrestha, "DC-SIGN gene promoter variants and IVIG treatment response in Kawasaki disease," Pediatric Rheumatology, vol. 11, no. 1, p. 32, 2013.

[14] A. Domínguez-Soto, E. Sierra-Filardi, A. Puig-Kröger et al., "Dendritic cell-specific ICAM-3-grabbing nonintegrin expression on M2-polarized and tumor-associated macrophages is macrophage-CSF dependent and enhanced by tumor-derived IL-6 and IL-10," The Journal of Immunology, vol. 186, no. 4, pp. 2192-2200, 2011.

[15] S. Lu, M. Bevier, S. Huhn et al., "Genetic variants in C-type lectin genes are associated with colorectal cancer susceptibility and clinical outcome," International Journal of Cancer, vol. 133, no. 10, pp. 2325-2333, 2013.

[16] Y. F. Xu, W. L. Liu, J. Q. Dong et al., "Sequencing of DC-SIGN promoter indicates an association between promoter variation and risk of nasopharyngeal carcinoma in Cantonese," BMC Medical Genetics, vol. 11, p. 161, 2010.

[17] E. Schaeffeler, U. M. Zanger, M. Eichelbaum, S. Asante-Poku, J. G. Shin, and M. Schwab, "Highly multiplexed genotyping of thiopurine s-methyltransferase variants using MALDTOF mass spectrometry: reliable genotyping in different ethnic groups," Clinical Chemistry, vol. 54, no. 10, pp. 1637-1647, 2008.

[18] L. Li, D. Liu, L. Hutt-Fletcher, A. Morgan, M. G. Masucci, and V. Levitsky, "Epstein-Barr virus inhibits the development of dendritic cells by promoting apoptosis of their monocyte precursors in the presence of granulocyte macrophagecolony-stimulating factor and interleukin-4," Blood, vol. 99, no. 10, pp. 3725-3734, 2002.

[19] M. Severa, E. Giacomini, V. Gafa et al., "EBV stimulates TLRand autophagy-dependent pathways and impairs maturation in plasmacytoid dendritic cells: implications for viral immune escape," European Journal of Immunology, vol. 43, no. 1, pp. 147-158, 2013.

[20] M. Mezger, M. Steffens, C. Semmler et al., "Investigation of promoter variations in dendritic cell-specific ICAM3grabbing non-integrin (DC-SIGN) (CD209) and their relevance for human cytomegalovirus reactivation and disease after allogeneic stem-cell transplantation," Clinical Microbiology and Infection, vol. 14, no. 3, pp. 228-234, 2008.

[21] H. G. Burke and E. E. Heldwein, "Crystal structure of the human cytomegalovirus glycoprotein B," PLoS Pathogens, vol. 11, no. 10, article e1005227, 2015.

[22] M. Backovic, R. Longnecker, and T. S. Jardetzky, "Structure of a trimeric variant of the Epstein-Barr virus glycoprotein B," Proceedings of the National Academy of Sciences of the United States of America, vol. 106, no. 8, pp. 2880-2885, 2009.

[23] J. J. Reimer, M. Backovic, C. G. Deshpande, T. Jardetzky, and R. Longnecker, "Analysis of Epstein-Barr virus glycoprotein
B functional domains via linker insertion mutagenesis," Journal of Virology, vol. 83, no. 2, pp. 734-747, 2009.

[24] L. M. Hutt-Fletcher, "Epstein-Barr virus entry," Journal of Virology, vol. 81, no. 15, pp. 7825-7832, 2007. 


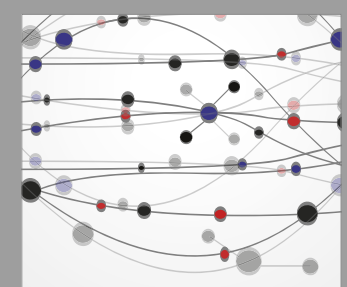

The Scientific World Journal
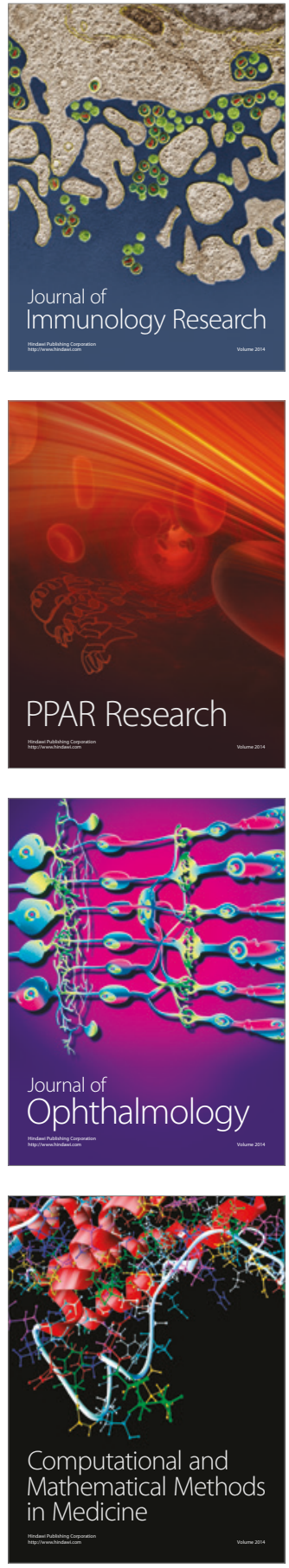

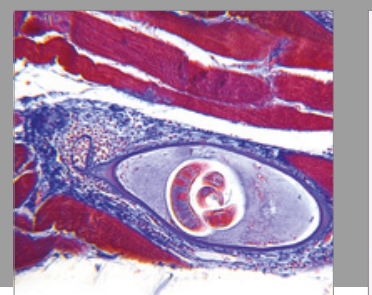

Gastroenterology Research and Practice
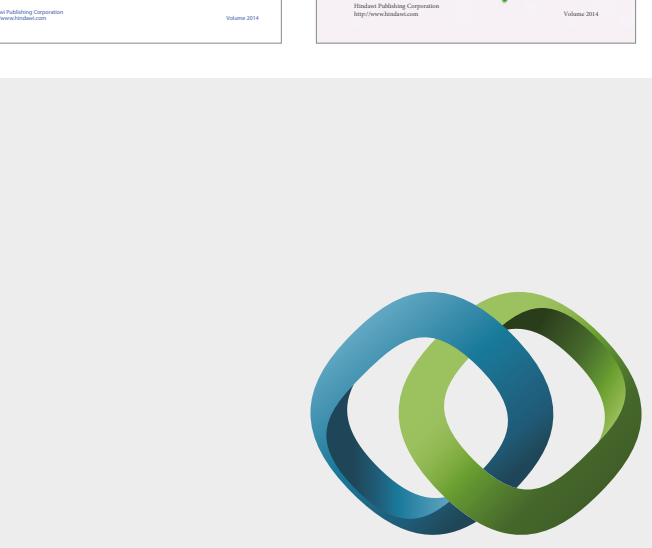

\section{Hindawi}

Submit your manuscripts at

https://www.hindawi.com
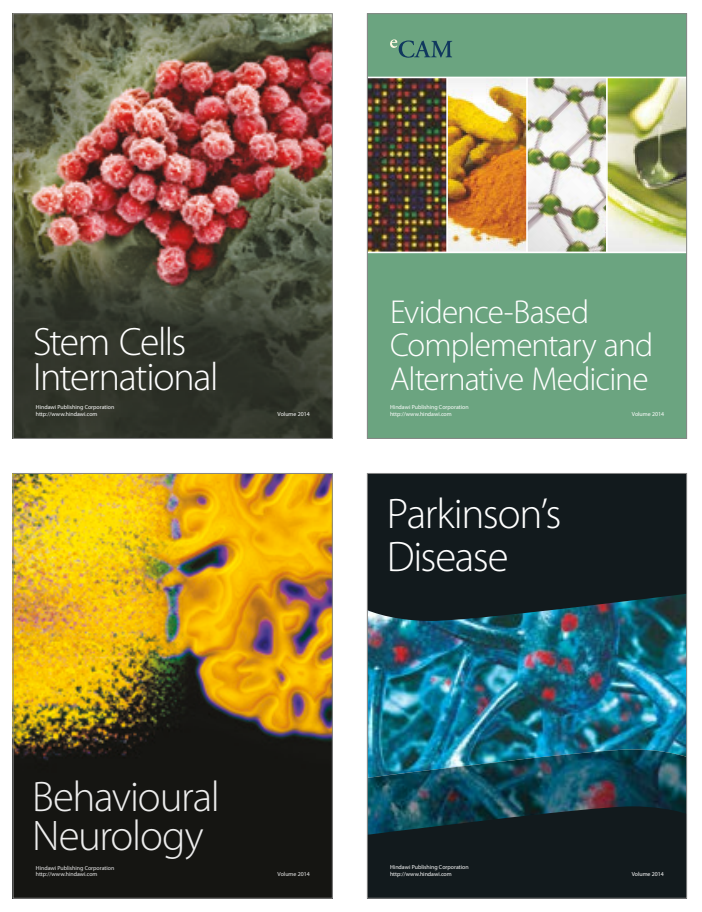
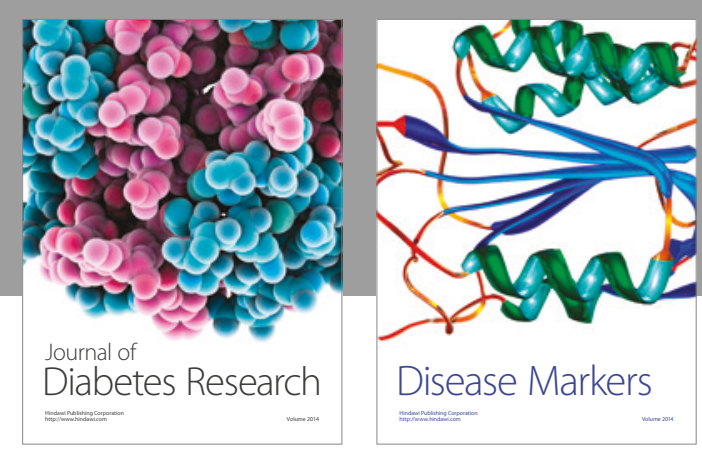

Disease Markers
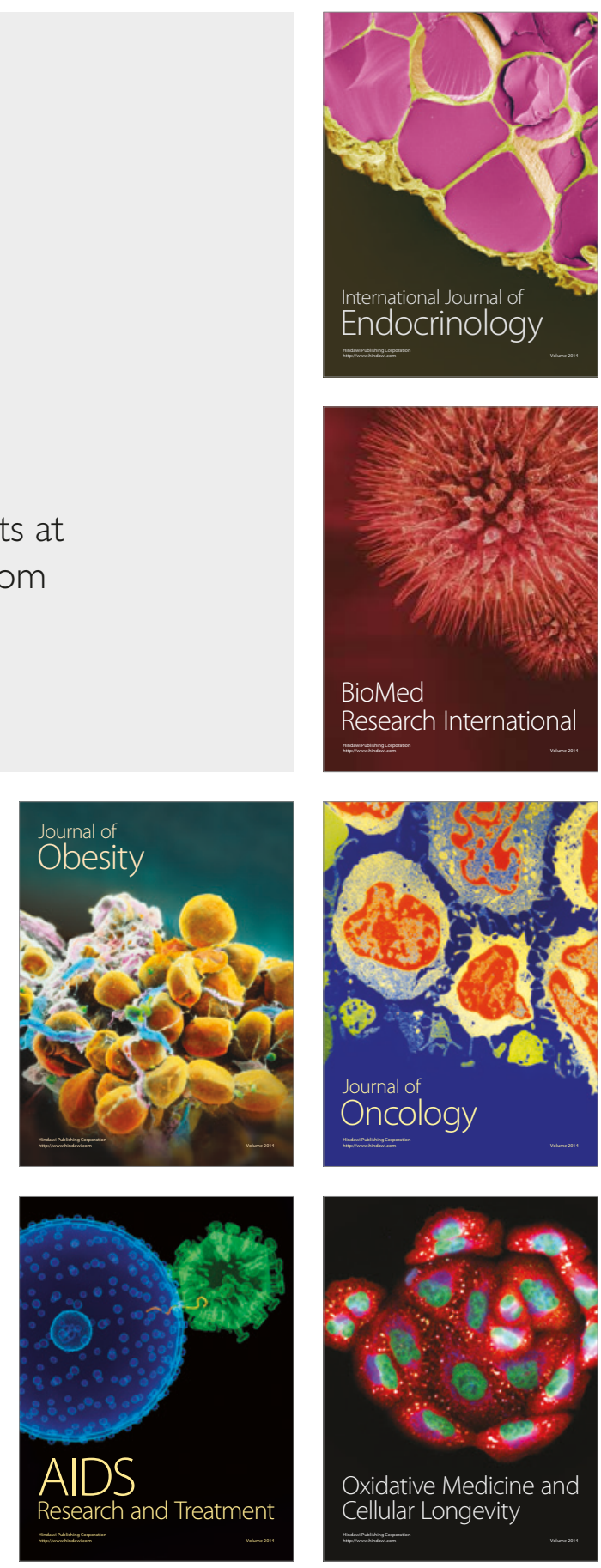